\title{
MS07-P15 | StRUCTURAL INSIGHT IN PEPTIDYL SUBSTRATE BINDING TO CYSTEINE
}

\section{CATHEPSINS}

Loboda, Jure (Institute Jozef Stefan, Ljubljana, SVN); Tusar, Livija (Jozef Stefan Institute, Ljubljana, SVN)

Jure Loboda ${ }^{1}$, Piotr Sosnowski ${ }^{2}$, Livija Tusar ${ }^{1,2}$, Robert Vidmar $^{1}$, Matej Vizovisek ${ }^{1}$, Jaka Horvat ${ }^{5}$, Gregor Kosec ${ }^{5}$, Francis Impens ${ }^{3,4}$, Hans Demol ${ }^{3}$, Boris Turk ${ }^{1}$, Kris Gevaert ${ }^{3,4}$, Dusan Turk ${ }^{1,2}$

1 Department of Molecular and Structural Biology, Jozef Stefan Institute, Jamova 39, Ljubljana, Slovenia

2 Centre of excellence CIPKEBIP, Jamova 39, Ljubljana, Slovenia

3 VIB Center for Medical Biotechnology, A. Baertsoenkaai 3, Ghent, Belgium

4 Department of Biomolecular Medicine, Ghent University, A. Baertsoenkaai 3, Ghent, Belgium

5 Acies Bio d.o.o., Tehnološki park 21, 1000 Ljubljana, Slovenia

We are trying to understand how cysteine cathepsins select their endogenous substrates. Analysis of proteomic study of the cell lysate, which was enriched with selected cathepsins, suggested representative peptides as a model of protein substrates based on cathepsin's specific cleavages. Using a structural approach we attempted to validate the peptide model with the crystal structures of active-site mutant human cathepsin $\mathrm{V}$ in complex with those peptides. The first generation peptides were designed to explore possible cooperative effects of amino acids binding into S1 and S2' subsites of the enzyme. The second generation peptides were hexapeptides selected from the proteomic data. We observed electron density of mainly cleaved or shifted peptides at the active site, possibly due to interactions of their charged termini. The analysis of the third generation peptides of various lengths, which have $\mathrm{N}$ - and $\mathrm{C}$ - termini protection, is ongoing. 